\title{
Multiple Primary Cancer and Survival after Lumpectomy with or without Radiotherapy for Ductal Carcinoma in Situ: A Propensity Score Matching Case-Control Study
}

Yitong Li ( $\sim 2204140421 @ c s u . e d u . c n)$

Second Xiangya Hospital https://orcid.org/0000-0002-4396-3080

Dengjie Ouyang

Second Xiangya Hospital

Qitong Chen

Second Xiangya Hospital

Tao Hong

Second Xiangya Hospital

Wenjun Yi

Second Xiangya Hospital

\section{Research Article}

Keywords: MPC, DCIS, radiotherapy

Posted Date: May 5th, 2021

DOl: https://doi.org/10.21203/rs.3.rs-463576/v1

License: (c) (1) This work is licensed under a Creative Commons Attribution 4.0 International License. Read Full License 


\section{Abstract}

Background: Radiotherapy combined with breast-conserving surgery is widely performed in patients with ductal carcinoma in situ (DCIS). This research was conducted to evaluate the use of radiotherapy to reduce the risk of MPC and mortality in DCIS patients.

Methods: 128,416 patients first diagnosed with DCIS between 1998 and 2015 who received lumpectomy in the Surveillance, Epidemiology, and End Results (SEER) 18 database were included. Information on age, race, year of diagnosis, laterality, pathologic grade, surgery, radiation, estrogen receptor status, progesterone receptor status, tumor size and vital status was extracted. A comparison of lumpectomy alone vs. lumpectomy plus radiotherapy was performed using 1:1 propensity score-based matching.

Results: Of the 128,416 patients with DCIS, 39,039 patients were treated with lumpectomy alone, and 69,377 were treated with lumpectomy and radiotherapy. The adjusted hazard ratio (HR) for death was 0.801 (95\%Cl: $0.7728-0.8301)$ and 0.7444 (95\% Cl: 0.7139-0.7761) for occurrence of MPC in the lumpectomy and radiotherapy vs. lumpectomy alone groups, respectively. Radiotherapy decreased the standardized incidence ratio (SIR) of MPC from 1.36 to 1.34. The incidence rate of a second breast cancer was reduced by $1.83 \%$ with radiotherapy (from $5.93 \%(2,177 / 36,688)$ to $4.10 \%(1,504 / 36,688)$ ) in the matched cohort.

Conclusion: Lumpectomy plus radiotherapy is associated with a significant reduction in mortality and risk of MPC, mainly second primary BC. Of those patients younger than 40 years, Black women with highgrade tumors were likely to benefit most. Radiotherapy reduced the risk of occurrence rather than the mortality of MPC patients to reduce the overall mortality.

\section{Introduction}

Ductal carcinoma in situ (DCIS) is a noninvasive condition in which abnormal cells are found in the lining of a breast duct ${ }^{[1]}$. Since the 1970s, multicenter prospective randomized controlled studies on breastconserving surgery for early breast cancer $(\mathrm{BC})$ patients have shown no significant difference in diseasefree and overall survival in such patients compared to those receiving total mastectomy ${ }^{[2,3]}$. In addition, radiotherapy plays an essential role in breast-conserving surgery ${ }^{[4,5]}$, resulting in low mortality for patients with $\mathrm{DCIS}{ }^{[6,7]}$. While long-term survivors benefit from systemic treatment, the frequency of multiple primary cancer (MPC) diagnosis after BC has been increasing, with MPC becoming one of the leading causes of death ${ }^{[8]}$.

Multiple primary cancer (MPC) is defined as more than 2 separate original tumors that simultaneously or successively occur in the same or different organs ${ }^{[9]}$. Previous studies reported a greater risk of MPC among patients with an initial diagnosis of breast cancer at the age of $<50$ years and in Black women with an ER-/PR- status than in Other patients ${ }^{[10]}$. A clinical study reported that the use of systemic therapy 
did not increase the risk of MPC ${ }^{[11]}$, while a nested case-control study using the SEER database suggested that radiotherapy $(H R=1.33)$ was related to the risk of second primary malignancy after $B C^{[12]}$.

To further study the risk of MPC and mortality in DCIS patients, we conducted a population-based study of women with DCIS using the Surveillance, Epidemiology, and End Results (SEER) database. We extracted data on age and year of diagnosis, laterality, pathologic grade, treatment (surgery and radiation), estrogen receptor (ER)status, progesterone receptor (PR) status, tumor size, MPC and death from $B C$. We sought to determine whether radiotherapy was associated with a reduced risk of $B C$ death and MPC in this cohort of women and to identify subgroups of women who might benefit the most from radiotherapy.

\section{Materials And Methods Study Population}

SEER 18 Regs Custom Data (with additional treatment fields), Nov 2017 Sub (1973-2015 varying) was used to identify the cohort of women for this study. We selected all female patients with confirmed stage $0 \mathrm{BC}$ coded by the 6 th edition breast-adjusted AJCC guidelines ${ }^{[13]}$. Among the cases classified as Tis, we excluded those associated with lobular carcinoma in situ, Paget's disease, and an unknown or invasive histology type. We excluded cases with unknown laterality and unknown or no surgical intervention for the primary tumor. We also excluded patients who underwent mastectomy to study those who underwent lumpectomy only. The flow chart of selection is provided in Fig. 1.

\section{Variables}

The SEER data included patient age at diagnosis, race (White, Black, or Other (American Indian/AK Native or Asian/Pacific Islander)), year of diagnosis, laterality, pathologic grade, surgery of the primary site, radiation, estrogen receptor status, progesterone receptor status, tumor size and vital status. We determined 2 time intervals: the time from DCIS to death and the time between the diagnosis of $\mathrm{BC}$ and a second primary cancer incidence. We divided all the patients diagnosed with a second primary BC after DCIS into ipsilateral and contralateral groups to compare the laterality of the two primary cancers. Patients eligible for study were categorized into 2 groups: lumpectomy without radiation and lumpectomy with radiation. The groups were compared for the variables above, and differences were evaluated using standardized differences.

\section{Matching}

Patients were 1:1 matched for age at diagnosis, race (White, Black, or Other (American Indian/AK Native or Asian/Pacific Islander)), year of diagnosis, laterality, pathologic grade, estrogen receptor status, progesterone receptor status and tumor size. Caliper matching was performed by matching participants 
who were within 0.2 times the standard deviation of their propensity score ${ }^{[14]}$. A standardized difference of greater than 0.1 was considered a meaningful imbalance between comparison groups. ${ }^{[15]}$

\section{Statistical Analysis}

Among all participants treated with lumpectomy, we conducted subgroup comparisons by age, race, ER status, PR status, tumor grade, and tumor size (using inverse probability of treatment weighting) to determine the extent to which radiation was associated with decreased risk of death in these various subgroups. The stabilized inverse probability of treatment-weighted estimates were truncated at the $1 \mathrm{st}$ and 99 th percentiles ${ }^{[15,16]}$.

The incidence of second primary cancer was compared to the expected incidence in the general population by calculating standardized incidence ratios (SIRs) and $95 \%$ confidence intervals (Cls). SIRs were calculated overall and stratified by clinical parameters. Cumulative survival curves for each patient variable were constructed using the Kaplan-Meier method and were analyzed using the log-rank test in GraphPad Prism 7. Variables with persistent prognostic value were identified by Cox proportional hazards regression, and the associated hazard ratios (HRs) were reported. Statistical analysis was performed using R version 3.6.3 software (http://www.r-project.org/).

\section{Results}

\section{Baseline Clinical Characteristics}

Among the 128,416 patients involved in the cohort, 39,039 patients were treated with lumpectomy alone, and 69,377 were treated with lumpectomy and radiotherapy (Table 1). Radiotherapy was performed with lumpectomy less often in elderly patients (older than 70 years old) than lumpectomy alone $(11,927$ [30.6\%] patients treated with lumpectomy and 12,351 [17.8\%] patients treated with lumpectomy plus radiotherapy). Slightly more Black patients and patients of Other races (American Indian/AK Native or Asian/Pacific Islander) received radiotherapy than radiotherapy plus lumpectomy (Black: 7,502 [10.8\%] vs. 3,958 [10.1\%]; Other races: 7,317 [10.5\%] vs. 3,823 [9.8\%]). The use of radiotherapy also increased with increasing tumor grade. Radiotherapy was more commonly used when the estrogen receptor status or the progesterone receptor status was positive.

Table 1. Baseline characteristics of patients with ductal carcinoma in situ 
Table 1

Baseline characteristics of patients with ductal carcinoma in situ

\begin{tabular}{|c|c|c|c|}
\hline & $N(\%)$ & & \\
\hline Value & Lumpectomy alone & Lumpectomy plus radiotherapy & SMD \\
\hline Patients & 39,039 & 69,377 & \\
\hline \multicolumn{4}{|c|}{ Age at diagnosis, y } \\
\hline$<40$ & $864(2.2)$ & $1,440(2.1)$ & \multirow[t]{4}{*}{0.303} \\
\hline $40-54$ & $11,943(30.6)$ & $25,388(36.6)$ & \\
\hline $55-69$ & $14,305(36.6)$ & 30,198 (43.5) & \\
\hline $70+$ & $11,927(30.6)$ & $12,351(17.8)$ & \\
\hline \multicolumn{4}{|l|}{ Race } \\
\hline Black & $3,958(10.1)$ & $7,502(10.8)$ & \multirow[t]{4}{*}{0.093} \\
\hline Other & $3,823(9.8)$ & $7,317(10.5)$ & \\
\hline Unknown & $520(1.3)$ & $343(0.5)$ & \\
\hline White & $30,738(78.7)$ & $54,215(78.1)$ & \\
\hline \multicolumn{4}{|l|}{ Year of diagnosis } \\
\hline 1998-2004 & $13,621(34.9)$ & $19,920(28.7)$ & \multirow[t]{3}{*}{0.133} \\
\hline 2005-2009 & $11,176(28.6)$ & $21,669(31.2)$ & \\
\hline 2010-2015 & $14,242(36.5)$ & $27,788(40.1)$ & \\
\hline Laterality = right & $19,033(48.8)$ & $33,932(48.9)$ & 0.003 \\
\hline \multicolumn{4}{|l|}{ Grade } \\
\hline I & $6,843(17.5)$ & $7,569(10.9)$ & \multirow[t]{4}{*}{0.35} \\
\hline II & $15,056(38.6)$ & $24,538(35.4)$ & \\
\hline III & $10,063(25.8)$ & $28,295(40.8)$ & \\
\hline Unknown & $7,077(18.1)$ & $8,975(12.9)$ & \\
\hline \multicolumn{4}{|c|}{ Estrogen receptor status } \\
\hline Negative & $2,329(6.0)$ & $7,128(10.3)$ & \multirow[t]{3}{*}{0.314} \\
\hline Positive & $20,459(52.4)$ & $43,114(62.1)$ & \\
\hline Unknown & $16,251(41.6)$ & 19,135 (27.6) & \\
\hline
\end{tabular}




\section{$N(\%)$}

\section{Progesterone receptor status}

\begin{tabular}{|c|c|c|c|}
\hline Negative & $3,914(10.0)$ & $11,372(16.4)$ & \multirow[t]{3}{*}{0.308} \\
\hline Positive & $17,244(44.2)$ & 35,899 (51.7) & \\
\hline Unknown & $17,881(45.8)$ & 22,106 (31.9) & \\
\hline \multicolumn{4}{|c|}{ Tumor size, cm } \\
\hline$<2.0$ & 22,195 (56.9) & $41,597(60.0)$ & \multirow[t]{4}{*}{0.143} \\
\hline $2.0-4.9$ & $4,481(11.5)$ & $9,951(14.3)$ & \\
\hline$\geq 5.0$ & $1,075(2.8)$ & $1,572(2.3)$ & \\
\hline Unknown & $11,288(28.9)$ & $16,257(23.4)$ & \\
\hline
\end{tabular}

\section{Radiotherapy Reduced The Risk Of Mortality And Mpc}

In the matched comparison of patients treated with lumpectomy vs. lumpectomy and radiotherapy, radiotherapy reduced the risk of death $(\mathrm{HR}=0.7444,95 \% \mathrm{Cl}: 0.7139-0.7761)$ (Fig. 2a). In addition, we determined the time interval between the first diagnosis of DCIS and the occurrence of multiple primary cancer (MPC) among the 36,688 propensity-matched pairs. The adjusted HR for MPC in the lumpectomy and radiotherapy vs. lumpectomy alone groups was 0.801 (95\% Cl: 0.7728-0.8301) (Fig. 2b). Moreover, radiotherapy benefited breast cancer specific survival.(Fig. s1)

\section{Younger Black women and high-grade tumors benefited the most from radiotherapy}

Next, we assessed the protective effect of radiotherapy on mortality and MPC incidence in different subgroups using inverse probability of treatment weighting (Fig. 3). All subgroups could benefit from radiotherapy, but the degree of benefit varied among different subgroups. For the risk of MPC, patients younger than 40 years benefited more than elderly patients $(0.783$ (95\% Cl: $0.685-0.895)$ vs. 0.802 (95\%Cl: 0.776-0.829)). Black women benefited more than White women ( 0.74 vs. 0.823$)$. The HR was 0.898 for patients with grade I tumors, 0.806 for patients with grade II tumors, and 0.793 for patients with high-grade tumors (grade III). The subgroup containing Black women younger than 40 years with highgrade tumors benefited more than other subgroups in terms of mortality (Fig. 3b). The only difference was that ER-positive patients benefited more than ER-negative in terms of a reduced risk of MPC $(0.777$ vs. 0.798 ), but the opposite was true in terms of mortality ( 0.65 vs. 0.555$)$. 


\section{Effect of radiotherapy on the standardized incidence ratio differed by tumor type}

Next, we calculated and compared the standardized incidence ratios (SIRs) among DCIS patients treated with lumpectomy vs. lumpectomy plus radiotherapy (Fig. 4). The rates of MPC that occurred at all sites among those treated with radiation (lumpectomy plus radiotherapy $\mathrm{SIR}=1.34,95 \% \mathrm{Cl}: 1.32-1.39$; lumpectomy alone $\mathrm{SIR}=1.36,95 \% \mathrm{Cl}$ : $1.34-1.38$ ) were lower than those among patients treated with other therapies, while both SIRs were higher than those for the general female population in the SEER database. The SIRs varied across different types of second primary cancer. For second BC, which accounted for the largest proportion of second primary cancer, the SIR was 2.11 (95\% Cl: $2.06-2.17)$ for patients treated with radiation and 2.19 (95\% Cl: $2.13-2.24)$ for patients treated without radiation. Radiotherapy had a protective effect on reducing the SIRs in patients with kidney, renal pelvis (1.18 to $1.14)$, colon and rectal cancer ( 0.83 to 0.82$)$, while the SIRs were higher in patients with oral cavity, pharynx cancer or splenic flexure cancer.

\section{Radiotherapy reduced both contralateral and ipsilateral second breast cancer incidence}

The incidence rate of second breast cancer was reduced by $1.83 \%$ with radiotherapy (from $5.93 \%$ $(2,177 / 36,688)$ to $4.10 \%(1,504 / 36,688))$ in the matched cohort. The incidence rate of contralateral second primary breast cancer incidence rate was reduced from $0.06 \%$ with radiotherapy (from $2.45 \%$ $(901 / 36,688)$ to $2.39 \%(879 / 36,688))$. The reduction was greater when the second breast cancer occurred ipsilaterally (from $3.48 \%(1,276 / 36,688)$ to $1.7 \%(625 / 36,688)$ ).

\section{Radiotherapy reduced the risk of occurrence of MPC rather than the mortality of MPC patients to reduce the overall mortality}

To elucidate the association between radiotherapy and the prognosis of patients with only one primary cancer, KM curve analyses were performed. MPC patients had a higher risk of death than patients with only one primary cancer, and the HR was 1.944 (95\% Cl: 0.848-2.045) (Fig. 5a). Radiotherapy reduced the risk of death in only the group of patients with one primary cancer (Fig. 5c), and no statistically significant reduction in the risk of death was found in the MPC group between patients treated with lumpectomy alone vs. those treated with lumpectomy plus radiotherapy.

\section{Discussion}

Our study is the first to characterize the risk of MPC and death in DCIS patients in a large population using propensity score matching analysis. Among those with DCIS treated with lumpectomy, the majority of patients received radiotherapy, and these patients were middle-aged and elderly and were more likely to 
have high-grade disease, ER-positive status, PR-positive status and smaller tumor size than those who did not undergo radiation (Table 1 ). These factors were accounted for in the matched analysis, and propensity scoring was used to balance the baseline characteristics between the groups (eTable 1). Therefore, we believe that the reductions in the risk of death and MPC are a result of the radiotherapy.

In the present study, the incidence of a second primary breast, kidney, renal pelvis and thyroid cancer as well as leukemia after DCIS increased, similar to previous studies ${ }^{[17,18]}$. As previously established, this increased risk of MPC is partially attributable to genetic and environmental influences. Importantly, we found that radiotherapy could reduce the risk of MPC by $20 \%$, and several reports support our conclusion. Kaire Innos et al ${ }^{[19]}$ concluded that the use of radiation therapy appears to be protective against the occurrence of subsequent ipsilateral invasive cancer after studying California Cancer Registry data on 23,547 women with DCIS first diagnosed in 1988-1999. Carolyn J. Kushner et al ${ }^{[20]}$ studied a dataset comprising 755 women with DCIS $(n=135)$ or stage I-II breast carcinoma $(n=620)$ and found that highdose radiotherapy (> 63 Grays) was not associated with a significantly increased risk of SMN. William Beaumont Hospital ${ }^{[21]}$ reported that the overall 10-year and 15-year rates of second malignancy among the surgery and radiotherapy ( + RT) and surgery alone cohorts were $14.2 \%$ and $24.2 \%$, and $16.4 \%$ and $22.6 \%$, respectively $(p=0.668)$. The 15 -year second contralateral $B C$ rate was $14.2 \%$ in the $S+R T$ cohort and $10.3 \%$ in the surgery alone cohort $(p=0.439)$. There are several possible underlying mechanisms: 1$)$ There is statistical evidence of departure from a linear dose-response, with a downward curvature seen at high doses, likely resulting from radiation-induced cell sterilization ${ }^{[22]}$. 2) High-dose radiation may suppress estrogen production by relevant target cells, as well as androgen production by other cells (with androgen having the potential to be converted peripherally into estrogen) ${ }^{[23]}$. 3) Previously hidden tumorassociated antigens, stress proteins and danger-associated molecular patterns will be exposed after radiotherapy ${ }^{[24]}$. The exposure of antigens might activate antitumor immunity ${ }^{[25]}$ and stimulate tumor cell killing.

In addition, we sought to identify those individuals who would benefit the most from radiotherapy. Lumpectomy combined with radiotherapy reduced the risk of both ipsilateral and contralateral second BC compared with lumpectomy alone. Among patients younger than 40 years, Black women with high-grade tumors were likely to benefit most. An interesting finding in our study is that patients with a negative ER status benefited more in terms of mortality, while ER-positive patients benefit slightly more in terms of the risk of MPC. Although data from endocrine therapy use are not included in the SEER database, it is likely that some ER-positive patients received hormone treatment combined with radiotherapy; thus, the mortality benefit from radiation appeared artificially low. In addition, the IDEAL trial (BOOG 2006-05) found that adjuvant endocrine therapy did not have a statistically significant effect on either overall survival or distant recurrence but reduced the risk of MPC $(H R=0.39,95 \% \mathrm{Cl}: 0.19-0.81$, log-rank $p=0.01)$ [26]. Considering the benefit of reducing the risks of mortality and MPC, we recommend that patients undergo radiotherapy whether receiving endocrine therapy or not. 
Several studies have pointed out the relationship between recurrence and mortality and the benefit of radiation. In RTOG 9804, a good-risk subset of patients with mammographically detected low- or intermediate-grade DCIS, measuring less than $2.5 \mathrm{~cm}$ with margins $\geq 3 \mathrm{~mm}$, the ipsilateral local failure rate was decreased significantly with the addition of radiotherapy ${ }^{[6]}$. In 2018 , Vasily Giannakeas et al studied 140,366 patients with DCIS diagnosed between 1998 and 2014 in the SEER 18 database. The adjusted HR for mortality associated with radiotherapy (based on 29,465 propensity-matched pairs) was 0.77 (95\% Cl: $0.67-0.88 ; \mathrm{P}<0.001)^{[27]}$. A recent population-based cohort study observed that the rate of ipsilateral invasive $\mathrm{BC}$ for women undergoing breast-conserving surgery with no record of radiotherapy was higher than that for women receiving radiotherapy (adjusted rate ratio $1.43,95 \%$ confidence interval 1.05 to 1.96), and the mortality from BC between the various treatment groups was similar ${ }^{[28]}$. In our study, we found that radiotherapy could reduce the risk of death, and the HR was $0.744(95 \% \mathrm{Cl}$ : $0.7139-$ 0.7761) among the cohort. However, the reduction in death only occurred in the patients with only one primary cancer, and radiotherapy did not have a statically significant effect among the MPC patients. We further compared mortality and found that the occurrence of MPC led to a higher risk of death than no occurrence of MPC. Therefore, radiotherapy reduced the risk of MPC rather than the death associated with MPC and thereby reduced overall mortality.

\section{Limitations}

Our study has several limitations. First, this is a retrospective study from the SEER database rather than a prospective cohort study, so inherent selection biases may undermine its external validity. Second, similar to other SEER database studies, this study lacks specific information on chemotherapy and hormone treatment. Third, the treatments for patients were not assigned at random. In addition, cases with some unknown variables were excluded; thus, the results may not include all patients with DCIS.

\section{Conclusion}

Among patients with DCIS, treatment with lumpectomy plus radiotherapy was associated with a significant reduction in mortality and risk of MPC, mainly second primary BC. Among patients younger than 40 years, Black women with high-grade tumors were likely to benefit most. The effect of the sequence of radiation and surgery and the form of radiotherapy should be explored in future studies.

\section{Declarations}

Author Contributions: Conceptualization, Yitong Li.; methodology, Yitong Li and Wenjun Yi.;; data curation, Yitong Li.; writing-original draft preparation, Yitong Li; writing-review and editing, Wenjun Yi.; visualization and supervision, Wenjun YI. All authors have read and agreed to the published version of the manuscript.

Funding: This research received no external funding. 
Conflicts of Interest: The authors declare no conflict of interest.

\section{References}

1. Cowell CF, Weigelt B, Sakr RA, Ng CK, Hicks J, King TA, Reis-Filho JS (2013) Progression from ductal carcinoma in situ to invasive breast cancer: revisited. Molecular oncology 7(5):859-869

2. Veronesi U, Cascinelli N, Mariani L, Greco M, Saccozzi R, Luini A, Aguilar M, Marubini E. Twenty-Year Follow-up of a Randomized Study Comparing Breast-Conserving Surgery with Radical Mastectomy for Early Breast Cancer. 2002, 347(16): 1227-1232

3. 13(4): $412-419$

4. Kunkler IH, Williams LJ, Jack WJL, Cameron DA, Dixon JM (2015) Breast-conserving surgery with or without irradiation in women aged 65 years or older with early breast cancer (PRIME II): a randomised controlled trial. The Lancet Oncology 16(3):266-273

5. Morrow M, Strom EA, Bassett LW, Dershaw DD, Fowble B, Giuliano A, Harris JR, O'Malley F, Schnitt SJ, Singletary SE, Winchester DP (2002) Standard for Breast Conservation Therapy in the Management of Invasive Breast Carcinoma. CA: A Cancer. Journal for Clinicians 52(5):277-300

6. McCormick B, Winter K, Hudis C, Kuerer HM, Rakovitch E, Smith BL, Sneige N, Moughan J, Shah A, Germain I, Hartford AC, Rashtian A, Walker EM, Yuen A, Strom EA, Wilcox JL, Vallow LA, Small W, Pu AT, Kerlin K, White J (2015) RTOG 9804: A Prospective Randomized Trial for Good-Risk Ductal Carcinoma In Situ Comparing Radiotherapy With Observation. J Clin Oncol 33(7)):709-715

7. van Maaren MC, de Munck L, de Bock GH, Jobsen JJ, van Dalen T, Linn SC, Poortmans P, Strobbe LJA, Siesling S (2016) 10 year survival after breast-conserving surgery plus radiotherapy compared with mastectomy in early breast cancer in the Netherlands: a population-based study. The Lancet Oncology 17(8):1158-1170

8. Effects of radiotherapy (2005) and of differences in the extent of surgery for early breast cancer on local recurrence and 15-year survival: an overview of the randomised trials. The Lancet 366(9503):2087-2106

9. Moertel CG, Dockerty MB, Baggenstoss AH (1961) Multiple primary malignant neoplasms. II. Tumors of different tissues or organs. Cancer 14:231-237

10. Silverman BG, Lipshitz I, Keinan-Boker L (2017) Second Primary Cancers After Primary Breast Cancer Diagnosis in Israeli Women, 1992 to 2006. Journal of global oncology 3(2):135-142

11. Yi M, Cormier JN, Xing Y, Giordano SH, Chai C, Meric-Bernstam F, Vlastos G, Kuerer HM, Mirza NQ, Buchholz TA, Hunt KK (2013) Other primary malignancies in breast cancer patients treated with breast conserving surgery and radiation therapy. Ann Surg Oncol 20(5):1514-1521

12. Li Z, Wang K, Shi Y, Zhang X, Wen J (2020) Incidence of second primary malignancy after breast cancer and related risk factors-Is breast-conserving surgery safe? A nested case-control study. Int J Cancer 146(2):352-362 
13. Goldstraw P, Chansky K, Crowley J, Rami-Porta R, Asamura H, Eberhardt WE, Nicholson AG, Groome P, Mitchell A, Bolejack V, International Association for the Study of Lung Cancer Prognostic Factors Committee S, Participating AB I, International Association for the Study of Lung Cancer S, Prognostic Factors Committee Advisory Participating B I. The IASLC Lung Cancer Staging Project: Proposals for Revision of the TNM Stage Groupings in the Forthcoming (Eighth) Edition of the TNM Classification for Lung Cancer. J Thorac Oncol 2016, 11(1): 39-51

14. Austin PC (2011) Optimal caliper widths for propensity-score matching when estimating differences in means and differences in proportions in observational studies. Pharmaceutical Statistics 10(2):150-161

15. Austin PC, Stuart EA (2015) Moving towards best practice when using inverse probability of treatment weighting (IPTW) using the propensity score to estimate causal treatment effects in observational studies. Stat Med 34(28):3661-3679

16. Cole SR, Hernan MA (2008) Constructing inverse probability weights for marginal structural models. Am J Epidemiol 168(6):656-664

17. An JH, Hwangbo Y, Ahn HY, Keam B, Lee KE, Han W, Park DJ, Park IA, Noh DY, Youn YK, Cho BY, Im SA, Park YJ (2015) A Possible Association Between Thyroid Cancer and Breast Cancer. Thyroid 25(12):1330-1338

18. Withrow DR, Morton LM, Curtis RE, Schonfeld SJ, Berrington de Gonzalez A (2017) Radiotherapy for ductal carcinoma in situ and risk of second non-breast cancers. Breast Cancer Res Treat 166(1):299-306

19. Innos K, Horn-Ross PL (2008) Risk of second primary breast cancers among women with ductal carcinoma in situ of the breast. Breast Cancer Res Treat 111(3):531-540

20. Kushner CJ, Hwang WT, Wang S, Solin LJ, Vapiwala N (2018) Long-term risk of second malignancies in women after breast conservation therapy for ductal carcinoma in situ or early-stage breast cancer. Breast Cancer Res Treat 170(1):45-53

21. Shaitelman SF, Grills IS, Kestin LL, Ye H, Nandalur S, Huang J, Vicini FA (2011) Rates of Second Malignancies After Definitive Local Treatment for Ductal Carcinoma In Situ of the Breast. International Journal of Radiation Oncology*Biology*Physics 81(5):1244-1251

22. Parveen B, Lene HSV, Cécile MR, Alice JS, Marilyn S, Susan AS, Rita W, Wendy L, Ann CM, Sue H, Debra LF, Joseph PN, Anna TM, Sarah SD, Charles AS, Leslie LR, Peter DI (2010) Risk of Second Primary Thyroid Cancer after Radiotherapy for a Childhood Cancer in a Large Cohort Study: An Update from the Childhood Cancer Survivor Study. Radiat Res 174(6a):741-752

23. Inskip PD (1994) Pelvic radiotherapy, sex hormones, and breast cancer. Cancer causes control: CCC 5(5):471-478

24. Vatner RE, Cooper BT, Vanpouille-Box C, Demaria S, Formenti SC (2014) Combinations of immunotherapy and radiation in cancer therapy. Frontiers in oncology 4:325

25. Suzuki R, Yoshida M, Oguchi M, Yoshioka Y, Tokumasu K, Osako T, Ono S, Ueno T, Miyagi Y (2020) Efficacy of radiation boost after breast-conserving surgery for breast cancer with focally positive, 
tumor-exposed margins. J Radiat Res 61(3):440-446

26. Blok EJ, Kroep JR, Meershoek-Klein Kranenbarg E, Duijm-de Carpentier M, Putter H, van den Bosch J, Maartense E, van Leeuwen-Stok AE, Liefers G-J, Nortier JWR, Rutgers EJT, van de Velde CJH (2018) Optimal Duration of Extended Adjuvant Endocrine Therapy for Early Breast Cancer; Results of the IDEAL Trial (BOOG 2006-05). JNCl: Journal of the National Cancer Institute 110(1):40-48

27. Giannakeas V, Sopik V, Narod SA (2018) Association of Radiotherapy With Survival in Women Treated for Ductal Carcinoma In Situ With Lumpectomy or Mastectomy. JAMA Netw Open 1(4):e181100

28. Mannu GS, Wang Z, Broggio J, Charman J, Cheung S, Kearins O, Dodwell D, Darby SC (2020) Invasive breast cancer and breast cancer mortality after ductal carcinoma in situ in women attending for breast screening in England, 1988-2014: population based observational cohort study. BMJ 369:m1570

\section{Figures}

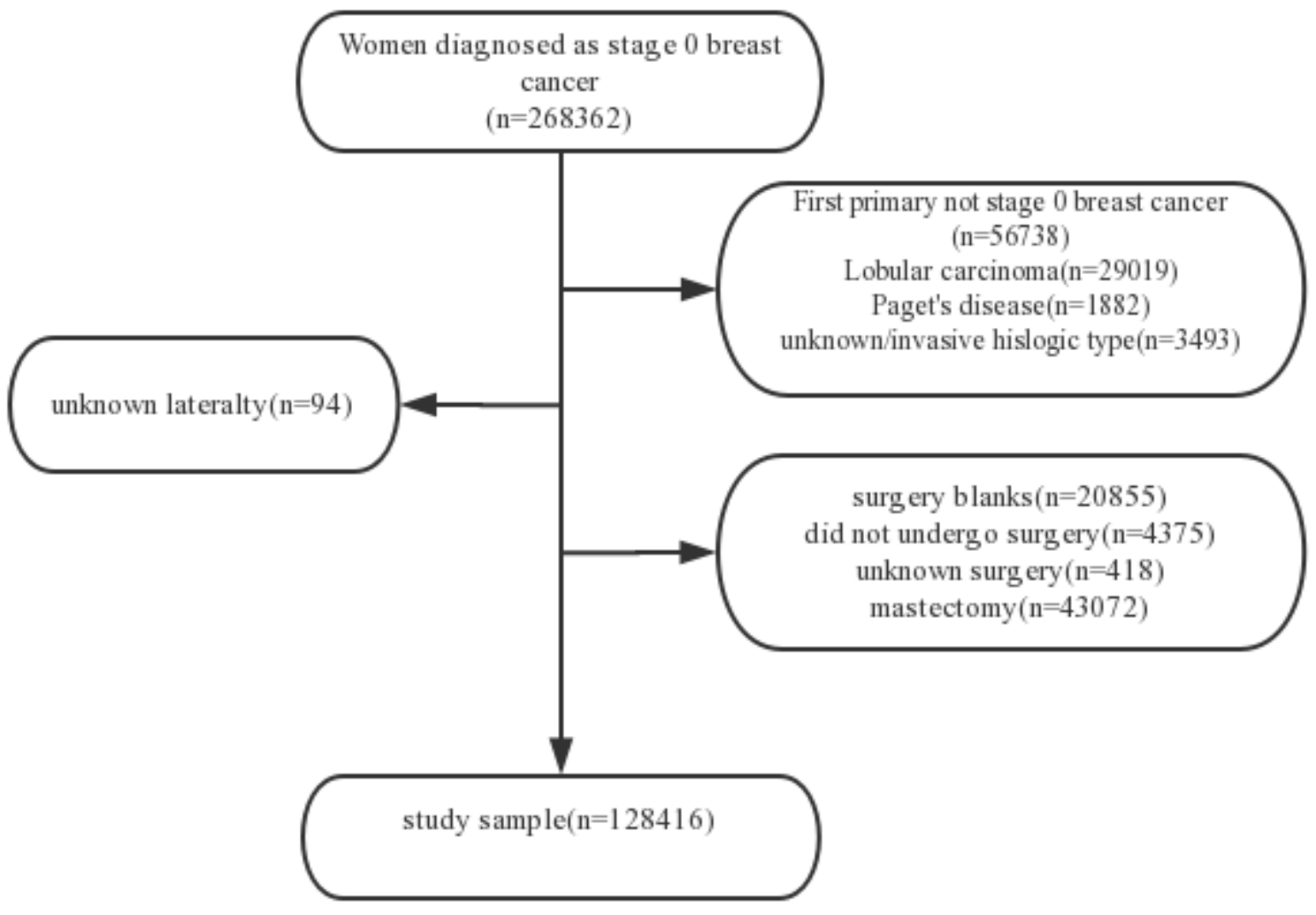

\section{Figure 1}

Flow chart for the selection of study samples 
A

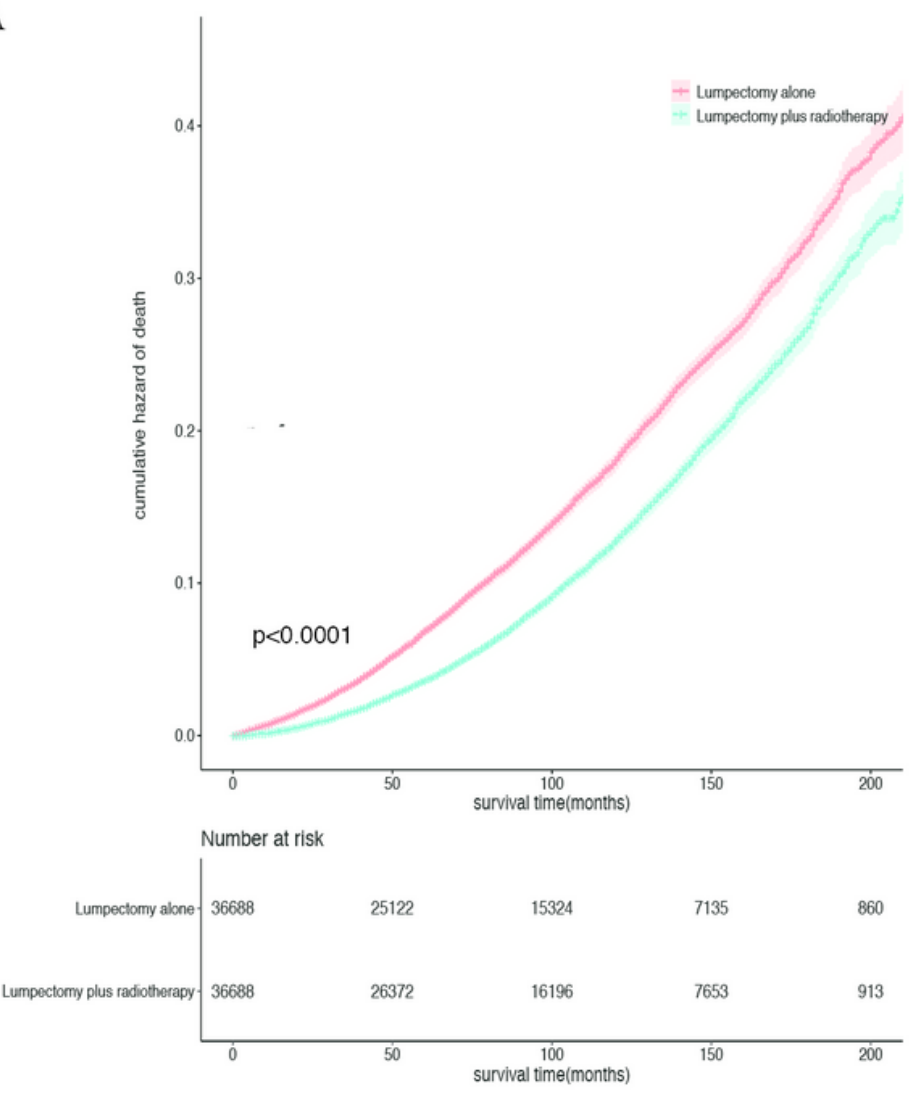

B

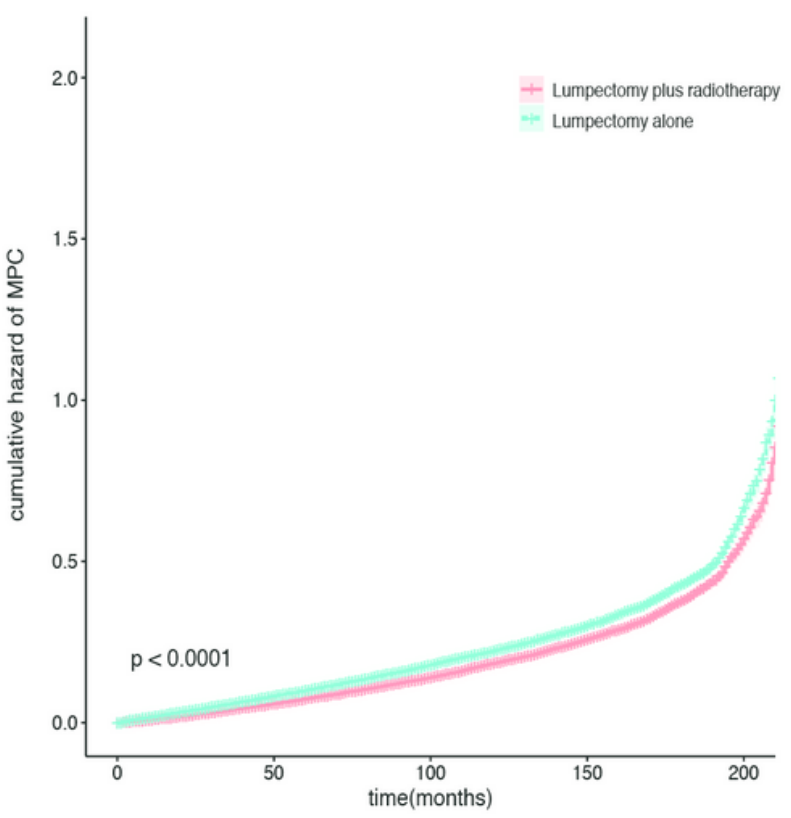

Number at risk

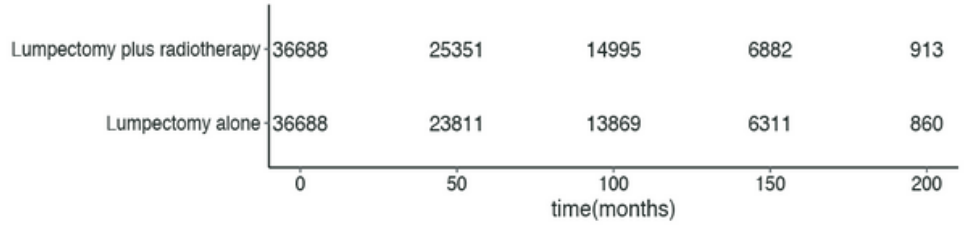

Figure 2

Kaplan-Meier curves of the cumulative hazard of death $(A)$ and the cumulative hazard of multiple primary cancer (B) for DCIS patients (all $p<0.0001$, log-rank test)
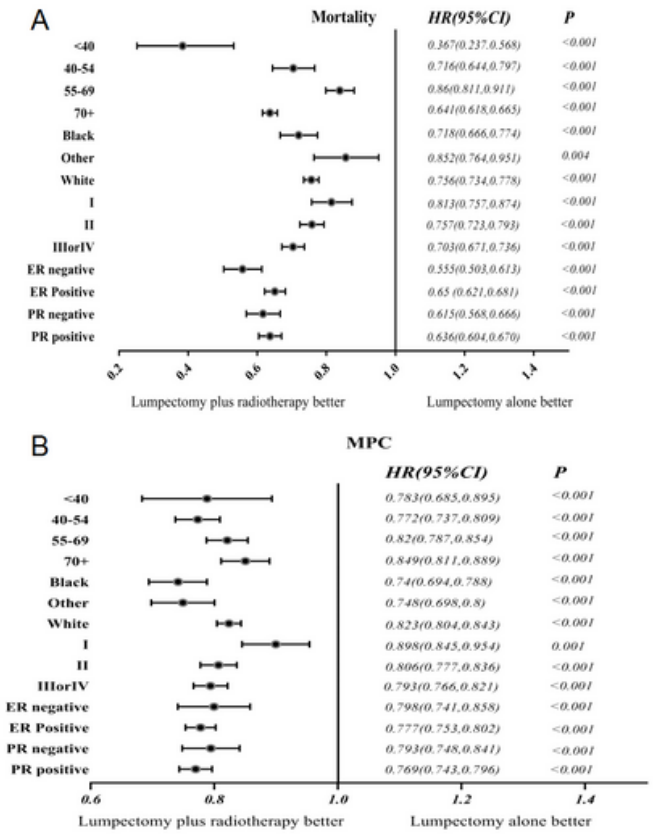
Figure 3

Hazard ratios for mortality $(A)$ and the risk of multiple primary cancer $(B)$ in various subgroups
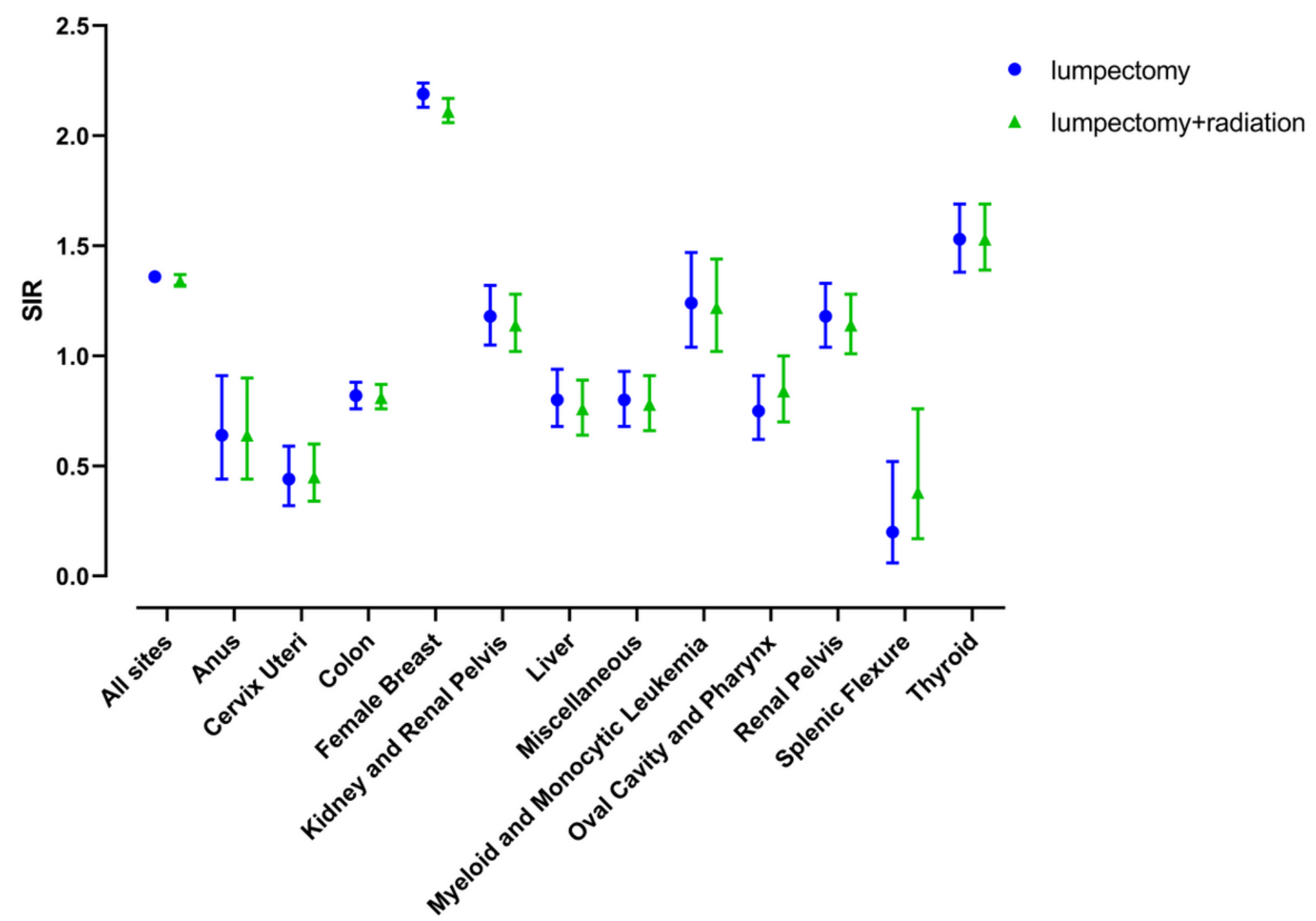

\section{Figure 4}

Standardized incidence ratios (SIRs) of second primary cancer after DCIS for patients treated with lumpectomy or lumpectomy plus radiation coded by "site recode B ICD-0-3/WHO 2008" showing a significant difference $(p<0.05)$ compared with those for the general population 

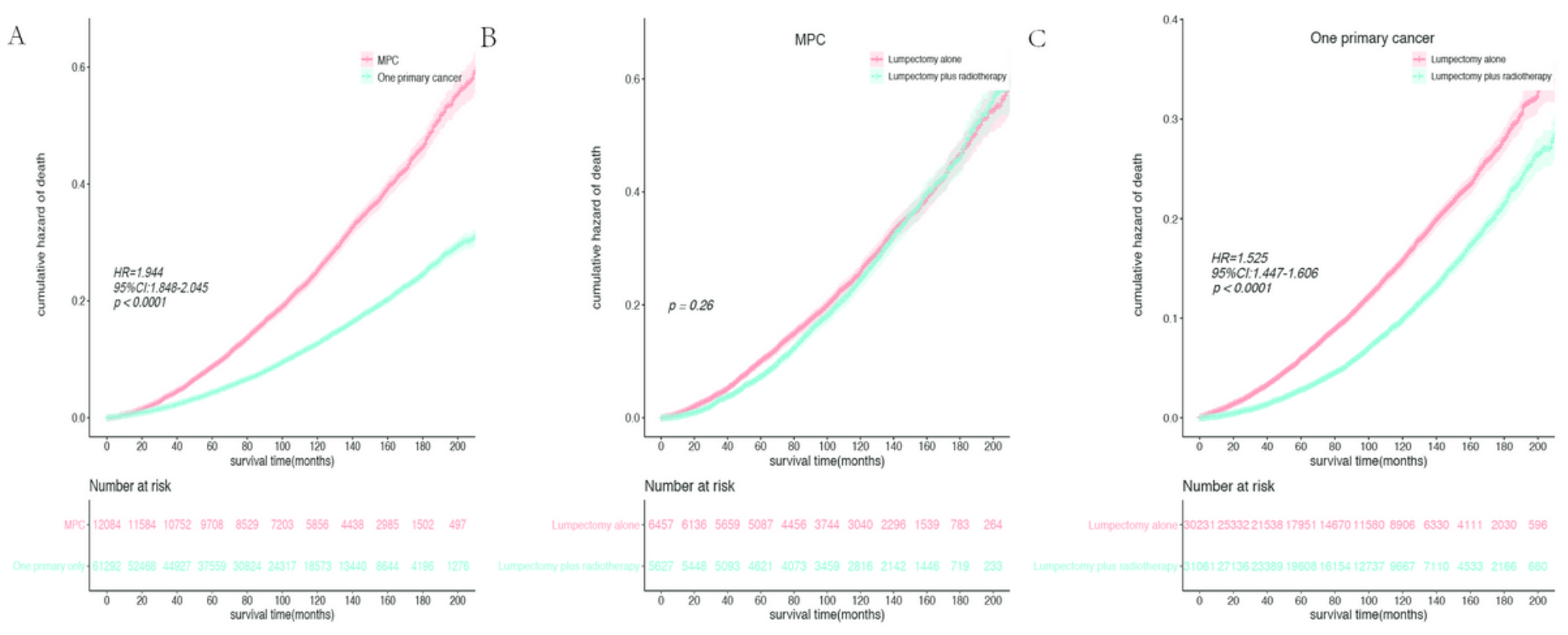

\section{Figure 5}

KM curves of patients overall (A); in the MPC group (B) and in the only one primary cancer group (C).

\section{Supplementary Files}

This is a list of supplementary files associated with this preprint. Click to download.

- Supplementary.docx 